\title{
26331 - GRANISETRON DOES NOT PREVENT NAUSEA AND VOMITING DURING C-SECTION
}

\section{Mrinalini Balki MD, Shilpa Kasodekar, MD; Sudhir Dhumne, MD; Jose Carvalho, Mount Sinai Hospital, Toronto, ONTARIO, Canada}

Introduction: Intraoperative nausea and vomiting (IONV) during Cesarean section (CS) under regional anesthesia remains a challenge in clinical practice. The etiologies of IONV include hypotension, vagal hyperactivity, visceral pain, intravenous opioids and uterotonics. The efficacy of prophylactic anti-emetics remains controversial. Different studies have shown their beneficial effects; however, the high incidence of IONV in their control groups suggests inadequate control of the multiple causative factors1. The purpose of our trial was to determine the efficacy of granisetron for prevention of IONV during CS under spinal anesthesia with strict control of the causative factors.

Methods: With REB approval, a prospective, randomized, double-blinded, placebocontrolled trial was conducted in 176 patients undergoing elective CS under spinal anesthesia. After preload with $10 \mathrm{ml} / \mathrm{kg}$ of lactated Ringer's solution, spinal anesthesia was administered with $0.75 \%$ hyperbaric bupivacaine $15 \mathrm{mg}$, fentanyl $10 \mu \mathrm{g}$ and morphine $0.1 \mathrm{mg}$. Aliquots of phenylephrine were used to maintain systolic blood pressure at $100 \%$ of baseline. Upon delivery of the fetus, oxytocin boluses of $0.5 \mathrm{IU}$ were administered as needed followed by maintenance infusion. The patients randomly received either granisetron $1 \mathrm{mg}$ or normal saline (placebo), intravenously, immediately after clamping of the umbilical cord. In case of persistent nausea or vomiting, rescue dimenhydrinate $50 \mathrm{mg}$ was administered intravenously. The primary outcome was the presence of postdelivery IONV. Secondary outcomes included need for rescue medication, hypotension, pain and nature of the surgical stimuli.

Results: There was no difference in maternal demographics and obstetric data between the groups. The overall incidence of postdelivery nausea and vomiting was $17 \%$ and $4 \%$ respectively. The incidence of IONV was similar in both groups. Other results are presented in the table.

Discussion: In contrast to the majority of studies in the literature1-3, our study shows that prophylactic granisetron $1 \mathrm{mg}$ is not effective in reducing the incidence and severity of IONV when the causative factors are strictly controlled. As compared to a previous study done at our institution4, our current study suggests that a higher dose of bupivacaine might result in more effective block thereby reducing visceral pain and thus IONV, especially when uterine exteriorization is performed.

References:

1. Int J Obstet Anesth 2005; 14: 230-4.

2. J Clin Anesth 2001;13: 430-5.

3. Anaesthesia 1999; 54: 479-82.

4. Anesthesiology 2005;102: SOAP A13 
GRANISETRON DOES NOT PREVENT NAUSEA AND VOMITING DURING CSECTION:

Outcome measures and intraoperative data

\begin{tabular}{lccc}
\hline Data & Granisetron & Placebo & p-value \\
& $\mathrm{N}=88$ & $\mathrm{~N}=88$ & \\
\hline Nausea (n, \%) & $16(18.2)$ & $14(15.9)$ & 0.69 \\
Retcling (n, \%) & $1(1.1)$ & $2(2.3)$ & 1.00 \\
Vomiting (n, \%) & $3(3.4)$ & $4(4.6)$ & 1.00 \\
Rescue antiemetic (n, \%) & $7(8)$ & $6(6.8)$ & 0.77 \\
Hypotension (n, \%) & $1(1.1)$ & $0(0)$ & 1.00 \\
Pain (n, \%) & $1(1.1)$ & $2(2.3)$ & 1.00 \\
Uterine exteriorization (n, \%) & $55(62.5)$ & $67(76.1)$ & 0.05 \\
Duration of uterine repair (min, meant:SD) & $11.13(4.5)$ & $11.69(4.8)$ & 0.43 \\
Tulbal ligation (n, \%) & $11(12.5)$ & $18(20.5)$ & 0.15 \\
Sensory block level (median, range) & T4 (T1-T7) & T4 (T1-T5) & 0.34 \\
\hline
\end{tabular}

